\title{
Quality of Service during Handover in Heterogeneous Wireless Network
}

\author{
Rashmi K.S \\ Mtech CS\&E, \\ VTU, PG Centre, \\ Mysore.
}

\author{
Mallappa D Mundavad \\ Mtech CS\&E, \\ VTU, PG Centre, \\ Mysore.
}

\author{
Shreedhar B Niradi \\ Mtech CS\&E, \\ VTU, PG Centre, \\ Mysore.
}

\begin{abstract}
In a cellular network, handoff is a process of transferring a mobile station (MS) from one base station (BS) or channel to another as the user is on the move. The handover process must take care of several parameters e.g. the handover scheme that is being used; number of free channels etc. The main objective of any handover scheme is to keep QoS up to the standard.

If handover scheme fails, it leads to disruption or termination of an ongoing call which is not desirable. The quality of any network is defined by its Quality of Service (QOS) parameters and handoff scheme plays an important role in maintaining the standards of QOS. In this paper, when the user is on the move, the handoff mechanism is analyzed on the basis of received signal strength from two different base stations and results are plotted using the Matlab code.
\end{abstract}

\section{General Terms}

Adaptive Received signal strength and handoff initiation time algorithm.

\section{Keywords}

Quality of Service, handover, handover-based traffic management, hotspot cell, adaptive RSS, traffic load, adaptive handover time.

\section{INTRODUCTION}

In next-generation wireless systems it is important for RRM functionality to ensure that the system in not overloaded and guaranteeing the needed requirements. If the system is not properly planned, the capacity is lower than required and the QoS is degraded. The system became overloaded and unstable. Therefore, a proper traffic management scheme is required to effectively manage overloaded traffic in the systems. In order to reduce interference between mobile stations (MS) and to increase number of subscribers in cellular phone network, reuse of frequency will be used, when MS moves between cells the uplink frequency and down link frequency must be changed according to the base stations frequencies to improve mobility in cellular phone so Hand off/Hand over technique will be used.

Handoff is the process of changing the channel (frequency, time slot, spreading code, or combination of them). Of course mobility is the most important facility in wireless cellular communication system. Usually, continuous service is achieved by supporting handoff (or handover) from one cell to another associated with the current connection while a call is in progress. It is often initiated either by crossing a cell boundary or by reducing the quality of the signal in the current channel. Poorly designed handoff schemes tend to generate very heavy signalling traffic, quality of service (QoS) will be decreased. Since the neighbouring cells are always using different frequency bands the handoffs are critical in cellular communication systems is that so negotiations must take place between the mobile station (MS), the current serving base station (BS), and the next base station. On the basis of currently known handoff schemes we need to design more general handoff algorithm which differently set the handoff threshold based on traffic of cells and the received signal strength of the target station with better performance in terms of probability of handoff delay and handoff failures In this thesis, a handover based traffic management algorithm to adaptively control the handover time according to the load status of cells is proposed. The traffic load can seriously affect on QOS for users, thus it requires efficient management in order to improve service quality. Hence the traffic load is considered as an important factor for initiating handover.

\section{ADAPTIVE RSS ALGORITHM TO CALCULATE THRESHOLD}

The main objective of this work is to design a handoverbased algorithm that adapts according to the load status of cells. A proper threshold value to control the handover initiation time according to the load status of cells, mobile's speed and handover types are used. The algorithm is designed to efficiently manage overloaded traffic in the cells and roll out the most precise or ideal time for handoff initiation.

In this paper a handover-based traffic management algorithm is proposed to adaptively control the handover time according to the load status of cells. First we develop a handover time algorithm, where in this proposed algorithm the threshold value is set based on traffic load of cells, speed of mobile terminal and distance parameters.

Terms used in the algorithm:-

Thres_serving:- The threshold value of the RSS to initiate the handover process. Therefore, when the RSS of SS drops below Thres_serving, the Mobile Switching Centre (MSC) registration procedures are initiated for MT's handover to TS.

Thres_min:- The minimum value of RSS required for successful communication between an MT and TS.

Thres_target:-The threshold value of RSS from target station for handoff execution.

Detailed Adaptive RSS algorithm is shown in the flow chart below. 


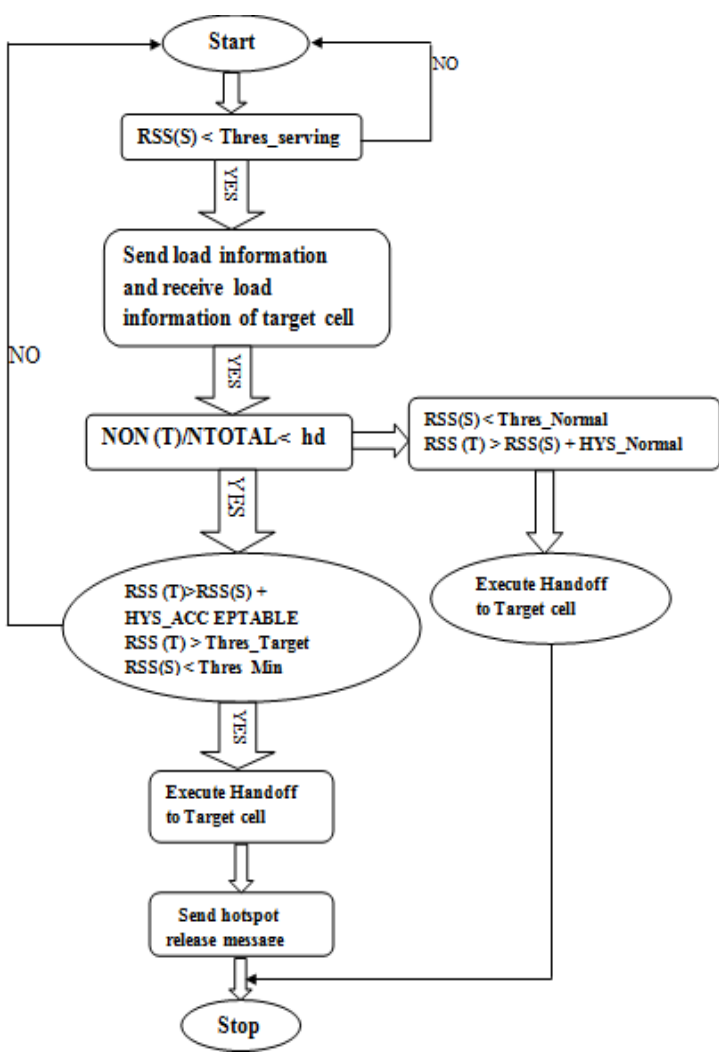

Fig 1: Flow chart of Adaptive RSS Algorithm

This algorithm is used for traffic distribution in the hotspot. Resources are shared among all users and the amount of available resources is determined by traffic load. If traffic load is concentrated in a cell, then that cell becomes hotspot cell. The hotspot cell can cause to block or drop calls and consequently degrades the service quality even though available resources are remained in neighboring cells. Therefore, it is essential to distribute traffic load of the hotspot cell in order to effectively use remained resources and maintain the acceptable service quality. Traffic load is the rate of channel occupancy in the cell. To maintain the quality of service and to make the effective usage of resources, distribution of traffic is needed.

In the proposed algorithm three states are considered, they are the HOLD and ON state between user and base station. The HOLD state has full downlink and thin uplink channel and ON state has both full downlink and uplink traffic channel. The load added by the handoff calls is defined as HANDOFF. The handoff call is assume to be in the ON state soon after the handoff process is over. The traffic load can be estimated by calculating the number of users in the three modes, HOLD, $\mathrm{ON}$ and HANDOFF which is described in the following equation 1 [3]

$$
\mathrm{NT}=\mathrm{NON}+\beta \mathrm{NHOLD}+\mathrm{NHO}
$$

Here NT is the amount of traffic loads,

$\mathrm{NON}$ is the number of users in the ON state,

NHOLD is the number of users in the HOLD state,

NHO is the number of handoff calls and

$\beta$ is an adaptive factor and the amount of traffic load varies from 0 to 1 .

The handover execution is executed not only by the measurement of signal strength but also the status of the traffic load where the proposed algorithm considering the load status of neighboring cells. These algorithms are executed when the neighboring cells not in the hotspot status. A slow handover time algorithm is used to delay time for the target cell to come out of the hotspot status and fast handover time algorithm is used when cell happens to be in hotspot status due to sudden occurrence of many new calls. Before accepting a new user, it requests the load information of the target cell in advance before handover execution.

The value of traffic load is approximated to 0 when the current cell is regarded as the lightly loaded cell and as the number of mobile nodes increase, the traffic load is taken as 1. The current cell becomes a hotspot. The hd value used in the algorithm is called hotspot threshold if the ratio of number of available resources by number of total resources is less than hd, then that cell have hotspot status.

When the RSS of the serving cell is less than threshold value, it sends the load information request message to the target cell and receive load information response message from the cell. The target cell calculates the amount of traffic load using Equation (1). If the amount of available resources of the target cell is less than the hotspot threshold, hd, the current serving cell sends hotspot alarm message to the target cell .Then, target cell completes the entire pending handover request \& send hotspot release message to serving station. Now, handover is executed to target station (TS). Proper threshold value should be carefully selected in order not to degrade the service quality of other users. An adaptive RSS threshold is recommended to use so that the mobile has enough time to initiate the handoff process.

\section{ADAPTIVE HANDOVER INITIATION TIME}

The Adaptive RSS algorithm can recognize the load status of the neighboring cells with the load information message in advance, before handover execution. After receiving the load information message, the proper threshold value should be carefully selected in order to initiate the handover process. In this algorithm, the mathematical equation used to control the handover time $\&$ thres_min according to the load status of cells, mobile's speed and handover signaling delay is

$$
\begin{aligned}
& P f=\operatorname{acos}(d / v) \text { atan }(a / 2 d) \text { - } \\
& P a=1-(1 / \pi)^{*} \Theta 1
\end{aligned}
$$

Equation (2) [8] and equation (3) [8] is used to calculate probability of handoff failure (pf) \& probability of false handoff initiation (pa) respectively. First, $d$ value for a desired value of pf using (1) is calculated, where d is MT's distance from the boundary of the serving BS, $\pi$ is the handoff signaling delay, a is cell size and $\Theta 1=a \operatorname{an}(\mathrm{a} / 2 \mathrm{~d})$.Then the required value of thres_min is selected by equation 4 .

$$
\text { thres_min= RSS (min)-10 log (a-d) + [db] }
$$

Equation 4 is derived from path loss model, where $\alpha$ is path loss coefficient. The thres_min value is should be carefully selected in order not to degrade the service quality of other users. Adaptive threshold value avoids too early or too late initiation of the handoff process (registration). They are completed before the user moves out of the coverage area of the serving network. 


\section{SIMULATION AND RESULTS 4.1. SIMULATION}

The simulation model is designed in MATLAB simulator. For simulations following scenarios and parameters are considered.

Hexagonal cell radius $(\mathrm{a})=1 \mathrm{~km}$

Max speed of mobile $(\mathrm{v})=100 \mathrm{~km} / \mathrm{h}$

Standard deviation of shadow fading $\varepsilon=8 d B$

Path-loss coefficient, $\alpha=4$

Minimum value of RSS $=-64 \mathrm{dBm}$

Thres_serving $=-74 \mathrm{~dB}$

Thres_target $=-79 \mathrm{~dB}$

Thres_normal $=-79 \mathrm{~dB}$

Hys_acceptable $=3 \mathrm{~dB}$

Hys_min $=0 \mathrm{~dB}$

Hys_normal $=2 \mathrm{~dB}$

Cell Capacity $=10$

\subsection{SIMULATION ENVIRONMENT}

In the simulation 7 hexagonal cells of radius 1 kilometer are considered. The cell capacity is taken to be 10. Mobile nodes will be moving at maximum speed of $100 \mathrm{kmph}$.The simulation of movement of mobile nodes in the hexagonal cells are shown below.

The red colored stars at the centre are the base stations to which the mobile nodes are connected

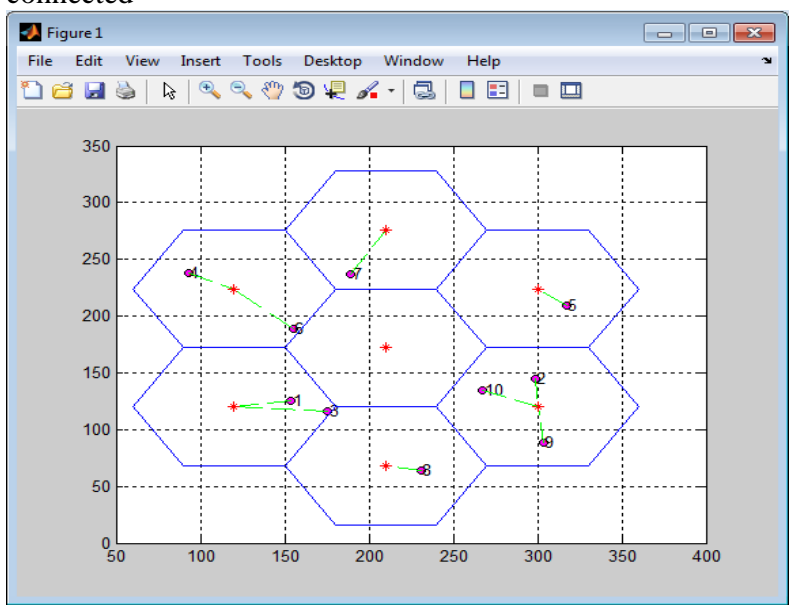

Fig 2: Mobility of nodes in the cells.

\subsubsection{False Handoff Initiation Probability variation with cell size}

It is clear from figure 3, as value of d increases, probability of false handoff initiation (pa) increases. This leads to unnecessary wastage of wireless resources. This also increases load on network as false handoff initiation takes place. We can see, as the cell size is decreasing, the pa is increasing. As in next generation the smaller cell size is required to increase capacity, it is very much important to select a proper value of $\mathrm{d}$, therefore Thres_serving, to reduce probability of false handoff initiation.

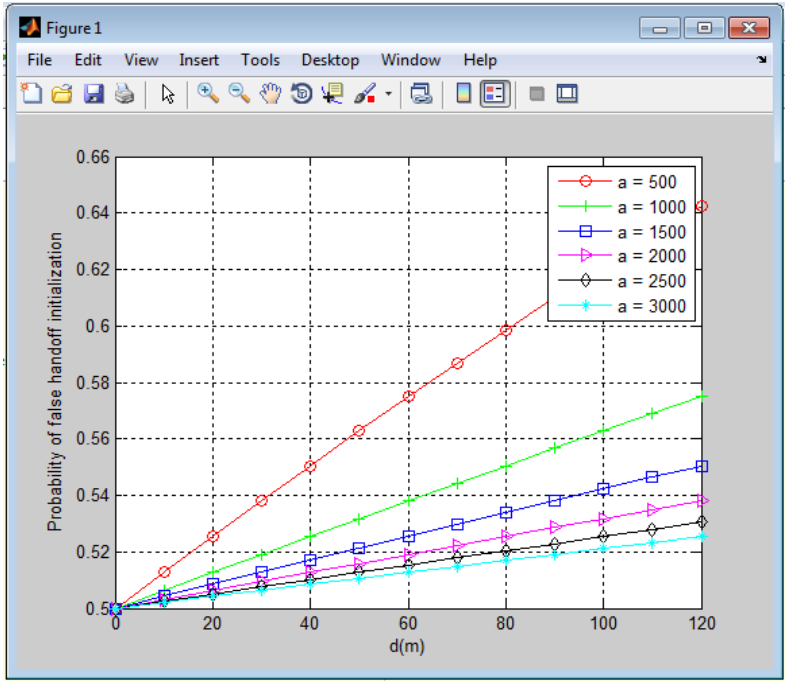

Fig 3: Relation between Handoff probability and $d$ with different value of cell size.

\subsubsection{Relation between probability of Handoff failure (pf) and Handoff signaling delay}

In order to analyze this, the velocity of vehicle is kept constant $100 \mathrm{~km} / \mathrm{hr}$ and result is analyzed by varying the signal delay. It has been observed in figure 4 as handoff latency increases, pf values increases for fixed value of thres_min .Higher the threshold value, lower will be the pf value. Therefore it is essential to predict the handoff delay in advance and then use an adaptive value of received signal to limit the handoff failure probability (pf) to a desired value. It is also observed that if proper signaling delay is used we can get desired pf value.

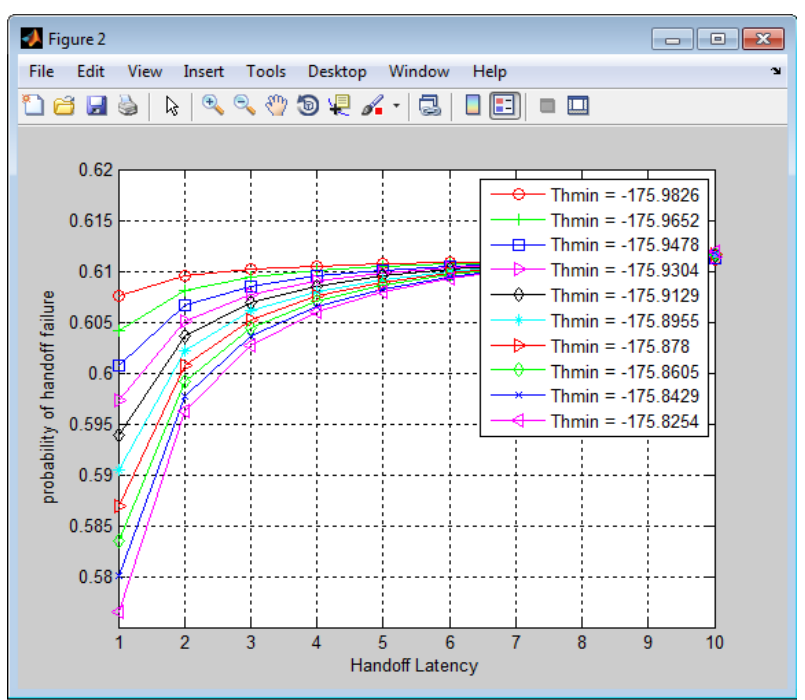

Fig 4: Relation between probability of handoff failure and handoff latency 


\subsubsection{Relation between Probability of Handoff Failure (pf) and Velocity}

Equation 2 shows, if a fixed value of thres_min is used (hence, a fixed value of corresponding $d$ ), the handoff failure probability depends on the speed of mobile terminal. The probability of handoff failure (pf) increases when MT's speed increases. As we can see in the figure $5 \mathrm{a} \& 5 \mathrm{~b}$, the value of $\mathrm{v}$ increases, for particular value of $\mathrm{d}$, pf increases for system handoff. This is because, as speed increases, MT requires less time to cover the region of serving station. The value of 3 represents intercellular handoff and $=1$ represents intracellular handoff. It is observed for larger value of d, pf values are smaller as compared to smaller values of $d$. This relation shows that the value of $d$,

Therefore the value of thres_min should be adaptive to the velocity of mobile terminal in order to have required probability of handoff failure (pf).

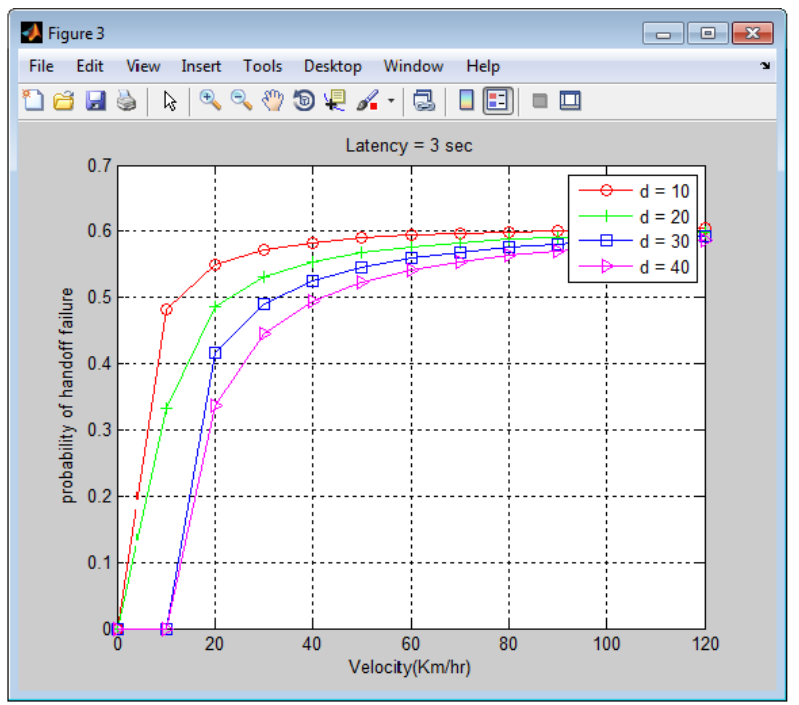

Fig 5a: Relation between handoff failure probability and velocity.

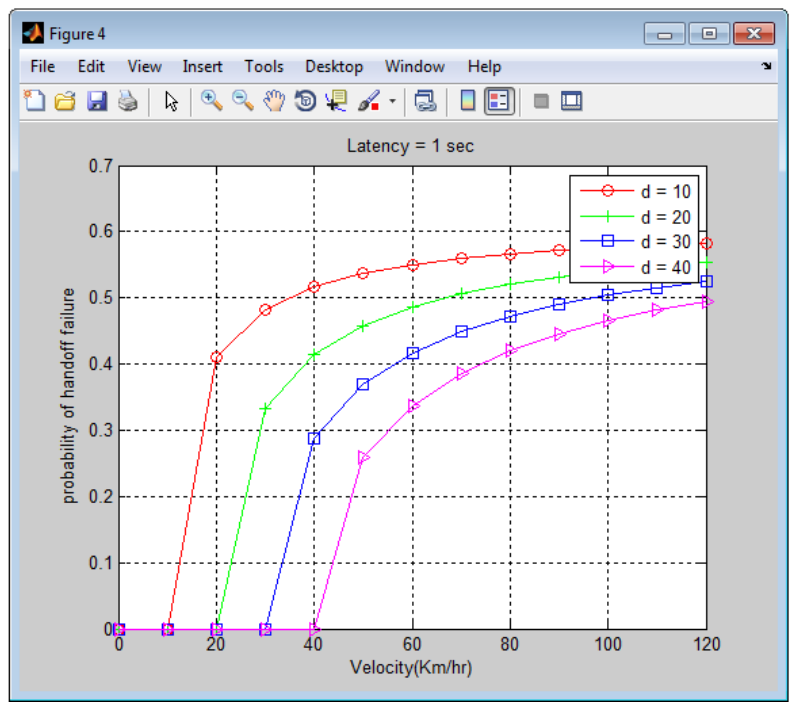

Fig 5b: Relation between handoff failure probability and velocity

\subsubsection{Relation between Probability of Handoff Failure (pf) and Handoff Signaling Delay}

Figure 6, shows handoff failure probability increases as hand off latency time increases. Therefore it is very important to select handoff type before and then use adaptive value of thres_min to fix particular handoff probability. High latency refers to intercellular handoff whereas low refers to intracellular handoff.

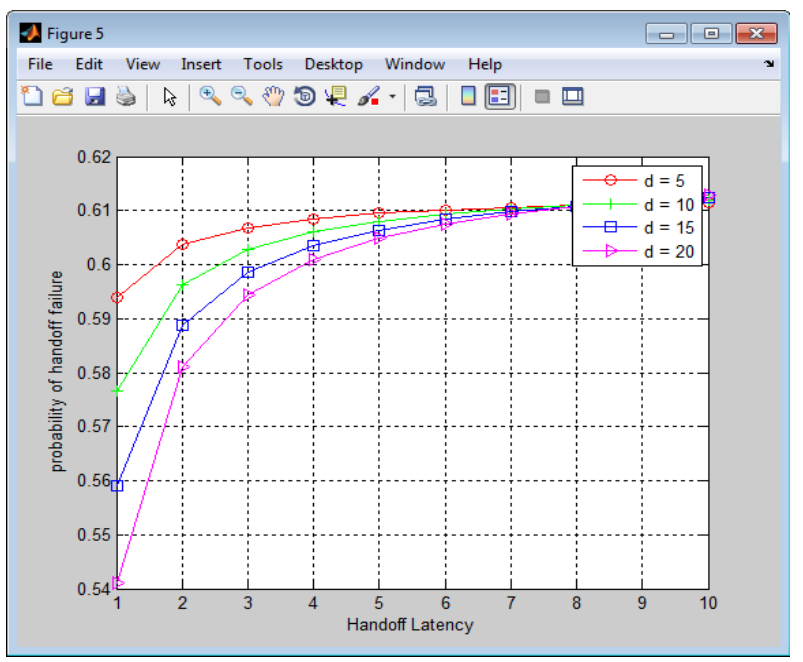

Fig 6: Relation between handoff failure probability and handoff latency

\subsubsection{Relation between Probability of Handoff Failure (pf) and Velocity for Different Value of Thres min}

In order to analyse this, the handoff latency is kept as 5 seconds and result is analysed by varying the velocity. It has been observed in figure 7 , as velocity increases, pf values increases for fixed value of thres min. Higher the threshold value, and lower will be the pf value. As we can see, for thres_min value of -175.2980 , even at the speed of $120 \mathrm{~km} / \mathrm{hr}$, the pf value lies between 0.1 to 0.2 therefore it is essential to know the velocity of mobile terminal and then use an adaptive value of received signal to limit the handoff failure probability (pf) to a desired value.

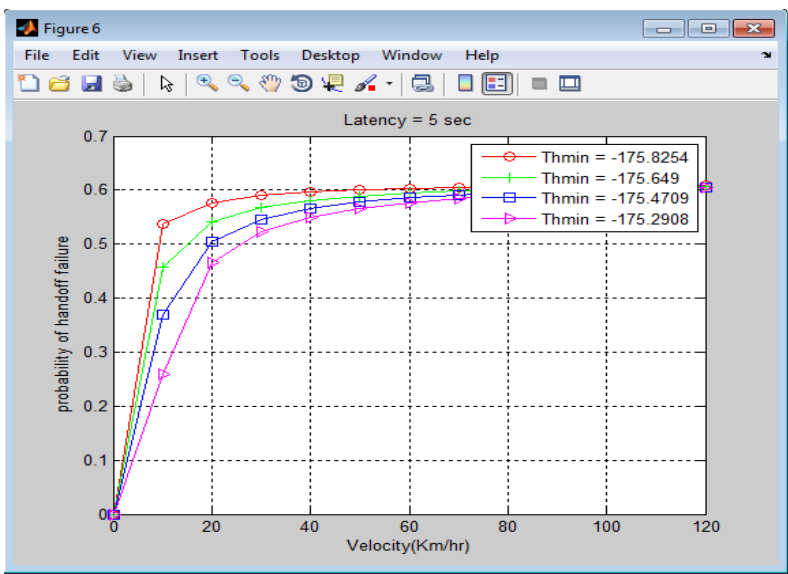

Fig 7: Relation between probability of handoff failure and velocity. 


\section{COMPARISONS BETWEEN PREVIOUS ALGORITHMS AND THE PROPOSED ALGORITHM}

In fixed RSS algorithm, fixed value of thres_min is used. It is calculated such that a user with highest speed is guaranteed the desired value of handoff failure probability (pf). Here desired value of pf is taken as 0.2 and handoff latency is taken as 1 second. In case of Adaptive RSS algorithm, according to the speed of mobile terminal, $d$ is calculated for desired handoff probability. With that calculated value of d, thres_min is calculated for handoff to take place. It is observed that with increase in velocity, pa for adaptive handoff algorithm increases whereas pa for fixed RSS algorithm remain constant, as it is calculated for maximum velocity of mobile terminal $(100 \mathrm{~km} / \mathrm{hr})$.After $100 \mathrm{~km} / \mathrm{hr}$, pa of Adaptive RSS crosses fixed one as we assume maximum speed in fixed RSS as $100 \mathrm{~km} / \mathrm{hr}$. For velocity values lesser than $100 \mathrm{~km} / \mathrm{hr}$, the pa value of adaptive RSS is always less than fixed one. We can see as in case of Adaptive algorithm thres_min is varying with velocity of mobile terminal. As velocity is increasing, thres_min value also increases, whereas in case of fixed one, the thres_ min is fixed. It shows that for high velocity MT, handoff initiation takes place earlier as compared to slow one. Thus thres_min is decided on the basis of velocity of mobile terminal and it leads to low hand drop call rate as compared to fixed RSS.

With respect to our previous and proposed algorithms, we have checked the simulation results of the effect of number of nodes, and the results are plotted as shown in the figures 8 and 9 below.

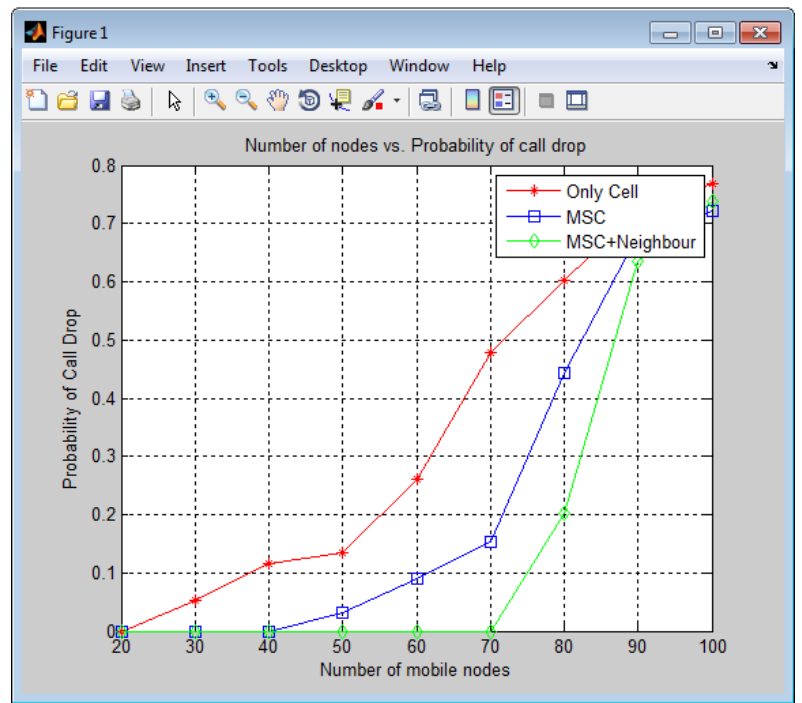

Fig 8: Number of nodes vs. probability of call drop

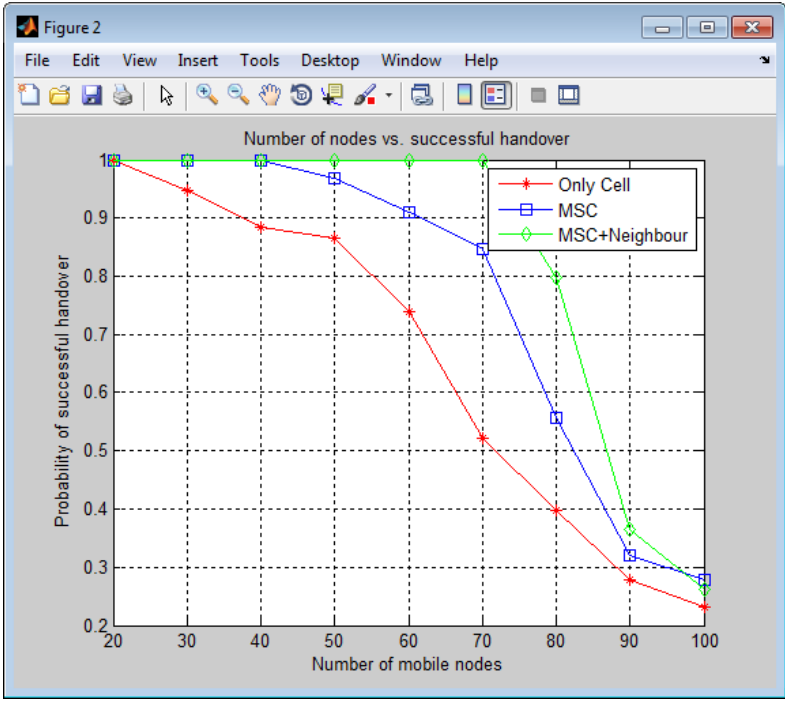

Fig 9: Number of nodes vs. Successful handover The effect of number of channels per cell is studied and the results are plotted as shown in the figures 10 and 11

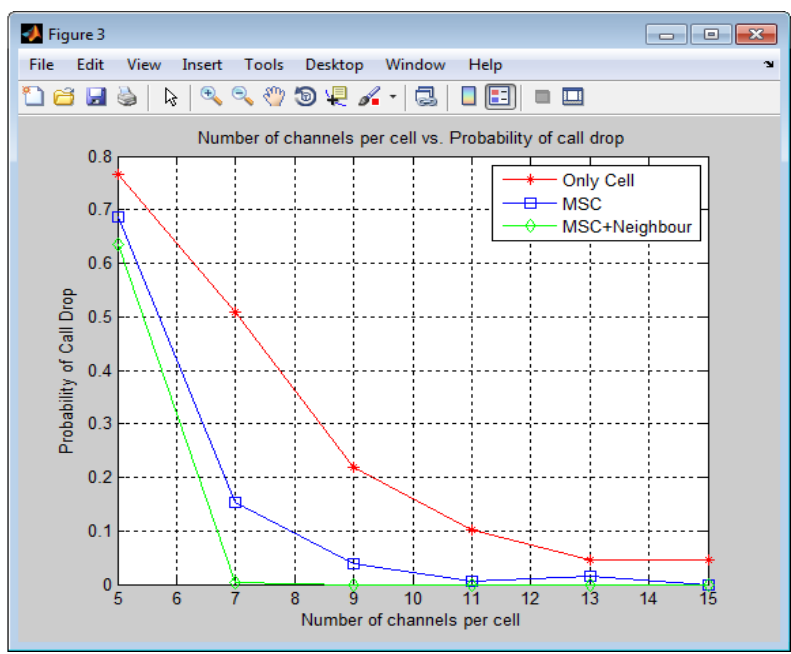

Fig 10: Number of channels per cell vs. Probability of call drop

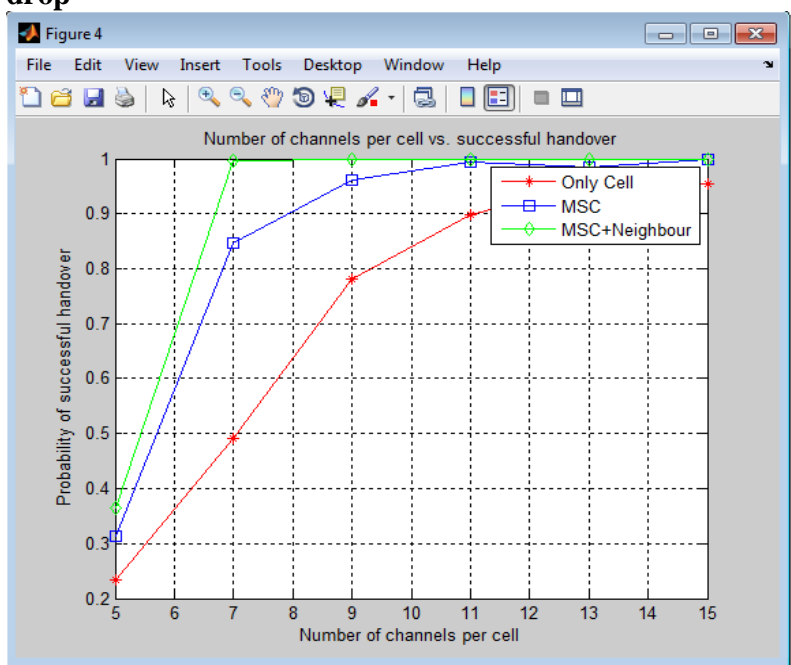

Fig 11: Number of channels per cell vs. Successful handover

The simulation generates the trace file as shown in the figure 12 with the performance matrices considered in our proposed algorithm showing the probability of handover and the 
probability of successful handover and the transfer of nodes from one base station to another.

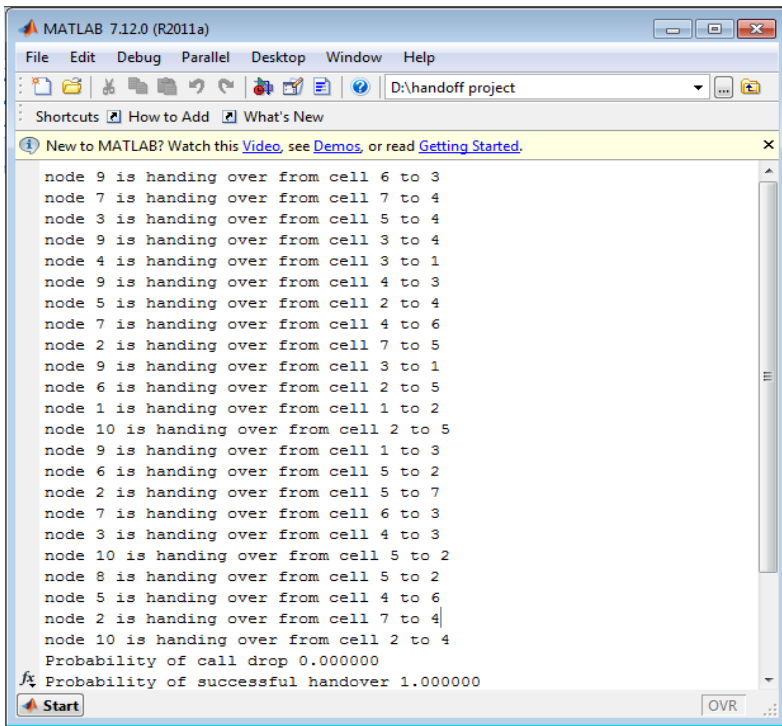

Fig 12: Trace file

\section{CONCLUSION AND FUTURE WORK}

Handover is one of the important concepts for mobility management in heterogeneous network. The primary goal of handoff is to allow user to access services while they keep on moving on in the network. In our paper, we have presented a handover-based algorithm that adapts according to the load status of cells. A proper threshold value to control the handover initiation time according to the load status of cells, mobile's speed and handover types are used. This algorithm is developed to efficiently manage overloaded traffic in the cells and roll out the most precise or ideal time for handoff initiation. Also, a comparison of probability of handoff failure in case of fixed RSS and Adaptive RSS algorithm has been shown. Results prove that a better QOS is achieved in Adaptive RSS than fixed RSS scheme. This algorithm would help in removing the problems like call failures, interruptions in data transfer etc and also it provides the base for future work in the area that is adaptive techniques in wireless network management.

\section{Future work}

The future work plan is to measure the system performance to improve handover latency and packet loss. Moreover, there is a plan to study a method to improve the service quality with respect to mobility management

\section{ACKNOWLEDGMENTS}

Our thanks to the Mr. Raghavendra K, Mtech (CS\&E), (PhD), Assistant Professor, who have helped towards development of this research paper.

\section{REFERENCES}

[1] Das S, Sen S \& Jayaram R, "A dynamic load balancing strategy for channel assignment using selective borrowing in cellular mobile environment," Proceedings of IEEE/ACM Conference on Mobile Computing and Networking (Mobicom96), pp. 73-84, 1996.

[2] I.F. Akyildiz, S. Mohanty, and J. Xie, "A Ubiquitous Mobile communication Architecture for Next Generation Heterogeneous Wireless systems," IEEE Radio Comm. Magazine, vol. 43, no. 6, pp. S29-S36, June 2005.

[3] Kim, D., Sawhney, M. \& Yoon, H., "An effective traffic management scheme using adaptive handover time in next generation cellular networks," Int. Journal of Network Management, pp. 139-154,2007

[4] Mark D. Austin and Gordon L. Stuber, "Velocity Adaptive Handoff Algorithms for Microcellular Systems," IEEE Trans. Veh. Tech., Vol. 43, No. 3, pp. 549-561, Aug. 1994.

[5] Salman A. AlQahtani, Ashraf S. Mahmoud and Asrar U. Sheikh, "Adaptive Radio Resource with Borrowing for Multi-Operators 3G+ Wireless Networks with Heterogeneous Traffic," Elsevier's Computer Communications Journal, vol. 29, Issue 15, pp. 29452951, 5 Sept. 2006.

[6] Bogdan Ciubotaru, Gabrie l-Miro Munte a n "Smooth Adaptive Soft Handover Algorithm for Multimedia Streaming over Wireless Networks" WCNC'09 Proceedings of the 2009 IEEE conference on Wireless Communications \& Networking Conference.

[7] Chen-Feng $\mathrm{Wu}$, Department of Information Management, Yu Da University, 168 “A Handoff Algorithm of Dynamic Decisions with Extension for Wirelesss Cellular Networks"

[8] "A RSS Based Adaptive Hand-Off Management Scheme In Heterogeneous Networks" Debabrata Sarddar, Shovan Maity, Arnab Raha, Ramesh Jana, Utpal Biswas, M.K. Naskar.

[9] D. Hong and S. S. Rappaport," Traffic model and performance analysis for cellular mobile radio telephone systems with prioritized and nonprioritized handoff procedures", IEEE Trans Vol. VT-35, No. 3, pp. 448461, August 1986

[10] Kil-Woong Jang, Ki-Jun Han "A Channel Assignment Scheme for Handoff in Wireless Mobile Networks" IJCA 2343, 2002

[11] Shantidev Mohanty and Ian F. Akyildiz,"A Cross-Layer (Layer $2+3$ ) Handoff Management Protocol for NextGeneration Wireless Systems", IEEE transactions on mobile computing, vol. 5, no. 10, October 2006.

[12] Dongwook Kim Dept. of Electr. Eng. \& Comput. Sci., Korea Adv. Inst. of Sci. \& Technol., Daejeon Namgi Kim ; Hyunsoo Yoon "Adaptive handoff algorithms for dynamic traffic load distribution in $4 \mathrm{G}$ mobile networks". 